\title{
MATHEMATIKA
}

A JOURNAL OF PURE AND APPLIED MATHEMATICS

Vol. 46, Part 1

June, 1999

No. 91

\section{ISOTROPIC SURFACE AREA MEASURES}

\section{A. GIANNOPOULOS AND M. PAPADIMITRAKIS}

Abstract. The purpose of this note is to bring into attention an apparently forgotten result of C. M. Petty: a convex body has minimal surface area among its affine transformations of the same volume if, and only if, its area measure is isotropic. We obtain sharp affine inequalities which demonstrate the fact that this "surface isotropic" position is a natural framework for the study of hyperplane projections of convex bodies.

\$1. Introduction. We shall work in $\mathbf{R}^{n}$ equipped with a fixed Euclidean structure and write $|\cdot|$ for the corresponding Euclidean norm. We denote the Euclidean unit ball and the unit sphere by $D_{n}$ and $S^{n-1}$ respectively, and we write $\sigma$ for the rotationally invariant probability measure on $S^{n-1}$. The volume of appropriate dimension will be also denoted by $|\cdot|$. We shall write $\omega_{n}$ for the volume of the Euclidean unit ball in $\mathbf{R}^{n}$. Finally, $L\left(\mathbf{R}^{n}, \mathbf{R}^{n}\right)$ is the space of all linear transformations of $\mathbf{R}^{n}$.

Let $K$ be a convex body in $\mathbf{R}^{n}$. The area measure $\sigma_{K}$ is defined on $S^{n-1}$ and corresponds to the usual surface measure on $K$ via the Gauss map. If $A$ is a Borel subset of $S^{\prime \prime-1}$, then

$$
\sigma_{\kappa}(A)=v\{x \in \text { bd }(K) \text { : the outer normal to } K \text { at } x \text { belongs to } A\},
$$

where $v$ is the $(n-1)$-dimensional surface measure on $K$. In the case where $K$ is a polytope with facets $\left\{F_{j}\right\} ; \leqslant m$ and normals $\left\{u_{j}\right\}, \leqslant m$, the measure $\sigma_{K}$ is purely atomic with mass $\left|F_{j}\right|$ at $u_{j}$, for $j=1, \ldots, m$. The surface area of $K$ is clearly given by

$$
\partial(K)=\sigma_{K}\left(S^{n-1}\right)
$$

For every convex body $K$ we define the affinely invariant quantity

$$
\hat{c}_{K}=\min \left\{\hat{c}(T K) /|K|^{(n-1) / n}: T \in L\left(\mathbf{R}^{n}, \mathbf{R}^{n}\right),|\operatorname{det} T|=1\right\},
$$

the minimal surface area of an affine image of $K$ with volume 1 . It is not hard to check that this minimum is attained for some $T$. 
Definition. A Borel measure $\mu$ on $S^{n-1}$ will be called isotropic if

$$
\int_{S^{n-1}}|\langle u, \theta\rangle|^{2} d \mu(u)=\frac{\mu\left(S^{n-1}\right)}{n}
$$

for every $\theta \in S^{n-1}$.

C. M. Petty [5] has proved the following characterization of the area measure of a convex body with minimal surface area.

TheOREM. 1. Let $K$ be a convex body in $\mathbf{R}^{n}$. Then $\partial(K)=\partial_{K}|K|^{(n-1) / n}$ if, and only if, $\sigma_{K}$ is isotropic.

In Section 2 we give a short proof of this fact. Actually, we came to the same conclusion without being aware of Petty's result. The latter was brought to our attention later, when V. D. Milman pointed out to us that Petty had studied the minimal surface position. The argument shows that for fixed volume the position is unique up to an orthogonal transformation.

We shall say that a convex body $K$ is surface isotropic if its area measure $\sigma_{K}$ is isotropic. This class of bodies with minimal surface area is very useful for questions related to hyperplane projections of convex bodies (one may say that it plays the role isotropic bodies - see [4] - play for questions related to sections). In Section 3 we demonstrate this in several ways.

We show that if $K$ is surface isotropic then

$$
\frac{\partial(K)}{2 n} \leqslant\left|P_{\theta}(K)\right| \leqslant \frac{\partial(K)}{2 \sqrt{n}}
$$

for every $\theta \in S^{n-1}$, where $P_{\theta}$ denotes the orthogonal projection onto the subspace $\theta^{\perp}$ perpendicular to $\theta$. Both estimates are sharp in the case of the cube. However, one can see that most projections of $K$ have area close to the upper bound. More precisely, there exists an absolute constant $c>0$ such that

$$
\left|P_{\theta}(K)\right| \geqslant c \frac{\partial(K)}{\sqrt{n}}
$$

with probability greater than $1-2^{-n}$ with respect to the standard measure $\sigma$ on $S^{n-1}$. This should be compared with the fact that all $(n-1)$-dimensional central sections of an isotropic body have the same area up to an absolute constant (see [4]).

The projection body $\Pi K$ of $K$ is the symmetric convex body whose support function is defined by $h_{\Pi K}(\theta)=\left|P_{\theta}(K)\right|, \theta \in S^{n-1}$. We write $\Pi^{*} K$ for the polar projection body.

The volume radius of $\Pi K$ and $\Pi^{*} K$ are determined by the minimal surface area $\partial_{K}:$ If $|K|=1$, then

$$
\left|\Pi^{*} K\right|^{1 / n} \simeq \frac{1}{\partial_{K}} \quad \text { and } \quad|\Pi K|^{1 / n} \simeq \frac{\partial_{K}}{n}
$$


up to absolute constants. This fact together with a result of $\mathrm{G}$. Zhang [11] show that $\partial_{K} \leqslant c n$ for every body $K$. K. Ball [2] has proved a reverse isoperimetric inequality of this type. His result is exact in the symmetric case: If $K$ is symmetric, then $\partial_{K} \leqslant 2 n$ and there is equality in the case of a cube.

$\mathrm{K}$. Ball notes in [1] that every convex body $K$ has an affine image $\widetilde{K}$ such that

$$
\left|P_{\theta}(\tilde{K})\right| \leqslant M \sqrt{n}|\tilde{K}|^{(n-1) / n}
$$

for some absolute constant $M>0$ and for every $\theta \in S^{n-1}$, and conjectures that the constant $M$ could be taken to be 1 in the symmetric case. Ball's reverse isoperimetric inequality and (1.1) show that, in the symmetric case, the surface isotropic position satisfies (1.4) with $M=1$. The cube shows that (1.4) is sharp.

K. Ball [1] has also proved that every convex body $K$ has an affine image $\tilde{K}$ such that, for every unit vector $\theta$,

$$
\left|P_{\theta}(\tilde{K})\right| \geqslant|\widetilde{K}|^{(n-1) / n} .
$$

The classical isoperimetric inequality and (1.2) show that with high probability the projections of a surface isotropic body satisfy

$$
\left|P_{\theta}(K)\right| \geqslant c|K|^{(n-1) / n} .
$$

The information given by (1.2) is actually much stronger. However, we do not know if (1.6) holds for every $\theta \in S^{n-1}$. We can show that, if $\sigma_{K}$ is isotropic, then the Loomis-Whitney type inequality

$$
|K|^{(n-1) / n} \leqslant \exp \left(\frac{1}{\partial(K)} \int_{S^{n-1}} \log \left|P_{\theta}(K)\right| \sigma_{K}(d \theta)\right)
$$

is true.

Finally, we show that extremal counterexamples for Shephard's problem (in the sense of [1]) can be constructed inside the class of surface isotropic symmetric convex bodies. There exist surface isotropic $K$ 's satisfying $\left|P_{\theta}(K)\right| \simeq|K|^{(n-1) / n}$ and others satisfying $\left|P_{\theta}(K)\right| \simeq \sqrt{n}|K|^{(n-1) / n}$ for every $\theta \in S^{n-1}$.

For well-known facts from the classical and the asymptotic theory of convex bodies we refer the reader to the books of R. Schneider [9] and G. Pisier [7].

\$2. A proof of Petty's Theorem. Petty's theorem will be an immediate consequence of the following three claims:

Cl.aim 1. Let $K$ be a convex body in $\mathbf{R}^{n}$ with $|K|=1$. If $\partial(K)=\partial_{K}$, then $\sigma_{K}$ is isotropic. 
A. GIANNOPOULOS AND M. PAPADIMITRAKIS

Proof. Let $R$ be a volume preserving transformation of $\mathbf{R}^{n}$. It is not hard to check that

$$
\partial\left(\left(R^{-1}\right)^{*} K\right)=\int_{S^{n-1}}|R(u)| \sigma_{K}(d u) .
$$

Consider any $T \in L\left(\mathbf{R}^{n}, \mathbf{R}^{n}\right)$ and let $\varepsilon>0$ be small enough. Then $(I+\varepsilon T) /$ $[\operatorname{det}(I+\varepsilon T)]^{1 / n}$ is volume preserving, so our minimality assumption for $K$ and (2.1) give

$$
\int_{S^{n-1}}|(I+\varepsilon T)(u)| \sigma_{K}(d u) \geqslant[\operatorname{det}(I+\varepsilon T)]^{1 / n} \partial_{K} .
$$

Observe that $|u+\varepsilon T u|=1+\varepsilon\langle u, T u\rangle+O\left(\varepsilon^{2}\right) \quad$ and $\quad[\operatorname{det}(I+\varepsilon T)]^{1 / n}=$ $1+\varepsilon(\operatorname{tr} T / n)+O\left(\varepsilon^{2}\right)$. Letting $\varepsilon \rightarrow 0$ we get

$$
\int_{s^{n-1}}\langle u, T u\rangle \sigma_{K}(d u) \geqslant \frac{\operatorname{tr} T}{n} \partial_{K},
$$

and by symmetry we conclude that

$$
\int_{S^{n-1}}\langle u, T u\rangle \sigma_{K}(d u)=\frac{\operatorname{tr} T}{n} \partial_{K}
$$

for every linear transformation $T$. This implies that

$$
\int_{S^{n-1}} u_{k} u_{l} \sigma_{K}(d u)=\frac{\partial_{K}}{n} \delta_{k l}, \quad k, l=1, \ldots, n,
$$

which completes the proof.

Claim 2. Let $K$ be a convex body in $\mathbf{R}^{n}$ with $|K|=1$. If $\sigma_{K}$ is isotropic, then $\partial(K)=\partial_{K}$.

Proof. Assume that $\sigma_{K}$ is isotropic. This is equivalent to the condition (2.4). If $T$ is any symmetric and positive definite volume preserving transformation, then

$$
\begin{aligned}
\partial(T K) & =\int_{S^{n-1}}\left|\left(T^{-1}\right)^{*} u\right| \sigma_{K}(d u) \\
& \geqslant \int_{s^{n-1}}\left\langle u, T^{-1} u\right\rangle \sigma_{K}(d u) \\
& =\frac{\operatorname{tr}\left(T^{-1}\right)}{n} \partial(K) \\
& \geqslant \partial(K),
\end{aligned}
$$


because $\operatorname{tr}\left(T^{-1}\right) / n \geqslant\left[\operatorname{det}\left(T^{-1}\right)\right]^{1 / n}=1$. This shows that $K$ has minimal surface area.

Claim 3. The minimal surface position is unique up to orthogonal transformations.

Proof. Assume that $K$ has minimal surface area, and that $\partial(R K)=\partial(K)$ for some volume preserving transformation $R$. We can write $R=U T$, where $T^{-1}$ is symmetric positive-definite and $U$ is an orthogonal transformation. Repeating the computation of (2.6) we have

$$
\partial(K)=\partial(U T(K))=\partial(T K) \geqslant \frac{\operatorname{tr}\left(T^{-1}\right)}{n} \partial(K) .
$$

This means that $\operatorname{tr}\left(T^{-1}\right)=n$, and since $T$ is symmetric positive-definite we must have $T=\mathrm{Id}$. This proves the claim.

We close this section with a lemma which is useful for approximation arguments.

LEMMA 2.1. Every surface isotropic convex body is the limit of a sequence of surface isotropic polytopes in the Hausdorff metric.

Proof. Let $K_{i}$ be a sequence of polytopes converging to $K$ in the Hausdorff metric. There exists a second sequence $T_{i}$ of volume preserving transformations such that $\tilde{K}_{i}=T_{i} K_{i}$ is surface isotropic. We may also assume that $T_{i} K_{i} \rightarrow L=$ $T K$ for some $T$ with $|\operatorname{det} T|=1$. Now $\sigma_{\tilde{K}_{i}} \rightarrow \sigma_{L}$ and one can easily check that $\sigma_{L}$ is isotropic. From Claim 3 it follows that $T \in O(n)$, and the proof is complete.

§3. Projections of a surface isotropic body. Let $K$ be a convex body in $\mathbf{R}^{n}$. The relation between the area of the $(n-1)$-dimensional projections of $K$ and the area measure $\sigma_{K}$ is well-known: For every $\theta \in S^{n-1}$ we have

$$
\left|P_{\theta}(K)\right|=\frac{1}{2} \int_{S^{n-1}}|\langle u, \theta\rangle| d \sigma_{K}(u) .
$$

Using (3.1) we can easily obtain lower and upper bounds for the projections of a surface isotropic body.

Proposition 3.1. If $\sigma_{K}$ is isotropic, then

$$
\frac{\partial(K)}{2 n} \leqslant\left|P_{\theta}(K)\right| \leqslant \frac{\partial(K)}{2 \sqrt{n}}
$$

for every $\theta \in S^{n-1}$. 
Proof. A direct application of the Cauchy-Schwarz inequality shows that

$$
\left|P_{\theta}(K)\right| \leqslant \frac{1}{2}\left(\int_{S^{n-1}}|\langle u, \theta\rangle|^{2} d \sigma_{K}(u)\right)^{1 / 2} \sqrt{\partial(K)}=\frac{\partial(K)}{2 \sqrt{n}} .
$$

On the other hand, it is obvious that

$$
\left|P_{\theta}(K)\right| \geqslant \frac{1}{2} \int_{S^{n-1}}|\langle u, \theta\rangle|^{2} d \sigma_{K}(u)=\frac{\partial(K)}{2 n} .
$$

The example of the cube $Q$ shows that both estimates in Proposition 3.1 are sharp. One can easily check that

$$
1 \leqslant\left|P_{\theta}(Q)\right| \leqslant \sqrt{n}
$$

for every $\theta \in S^{n-1}$ with equality on both sides for suitable $\theta$. The cube is surface isotropic, and $\partial(Q)=2 n$. Therefore, the ratio between the maximal and the minimal projection of a surface isotropic body can be as large as $\sqrt{n}$. However, we are going to show that the area of the projections is close to the upper bound with high probability. To this end, we use the following result of $\mathrm{K}$. Ball [2], whose proof is based on the Brascamp-Lieb inequality.

LEMMA 3.2. Let $\left\{u_{j}\right\}_{j \leqslant m}$ be unit vectors in $\mathbf{R}^{n}$ and $\left\{c_{j}\right\}_{j \leqslant m}$ be positive numbers satisfying

$$
\mathrm{Id}=\sum_{j=1}^{m} c_{j} u_{j} \otimes u_{j}
$$

Define a norm in $\mathbf{R}^{n}$ by $\|x\|=\sum_{j=1}^{m} \alpha_{j}\left|\left\langle x, u_{j}\right\rangle\right|$, where $\left.\alpha_{j}\right\rangle 0$. If $L$ is the unit ball of $\left(\mathbf{R}^{n},\|\cdot\|\right)$, then

$$
|L| \leqslant \frac{2^{n}}{n !} \prod_{j=1}^{m}\left(\frac{c_{j}}{\alpha_{j}}\right)^{c_{j}}
$$

Assume that $K$ is a polytope with facets $F_{j}$ and normals $u_{j}$, for $j=1, \ldots, m$, which has isotropic area measure. Then, (2.4) is equivalent to

$$
\mathrm{Id}=\sum_{j=1}^{m} \frac{n\left|F_{j}\right|}{\partial(K)} u_{j} \otimes u_{j}
$$

for every $x \in \mathbf{R}^{n}$. If $\Pi^{*} K$ is the polar projection body of $K$, we have

$$
\|\theta\|_{\Pi^{*} K}=\left|P_{\theta}(K)\right|=\frac{1}{2} \sum_{j=1}^{m}\left|F_{j}\right|\left|\left\langle\theta, u_{j}\right\rangle\right|
$$

for every $\theta \in S^{n-1}$. We can therefore apply Lemma 3.2 to obtain

$$
\left|\Pi^{*} K\right| \leqslant \frac{2^{n}}{n !}\left(\frac{2 n}{\partial(K)}\right)^{n}
$$


Approximating we get the same estimate for a general surface isotropic convex body. On the other hand, an application of Hölder's inequality shows that

$$
\left|\Pi^{*} K\right|=\omega_{n} \int_{S^{n}} \frac{1}{\left|P_{\theta}(K)\right|^{n}} \sigma(d \theta) \geqslant \omega_{n}\left(\int_{S^{n-1}}\left|P_{\theta}(K)\right| \sigma(d \theta)\right)^{-n} .
$$

Combining this with Cauchy's surface area formula

$$
\int_{S^{n-1}}\left|P_{\theta}(K)\right| \sigma(d \theta)=\frac{\omega_{n-1}}{n \omega_{n}} \partial(K),
$$

we have a double-sided estimate for the volume of the polar projection body of a surface isotropic body $K$.

Proposition 3.3. Let $K$ be a convex body in $\mathbf{R}^{n}$ whose area measure $\sigma_{K}$ is isotropic. Then

$$
\omega_{n}\left(\frac{n \omega_{n}}{\omega_{n-1}}\right)^{n} \frac{1}{\partial(K)^{n}} \leqslant\left|\Pi^{*} K\right| \leqslant \frac{4^{n} n^{n}}{n !} \frac{1}{\partial(K)^{n}} .
$$

Using the Blaschke-Santaló inequality and its exact reverse for zonoids (see [3], [8]) we estimate the volume of $\Pi K$ in terms of $\partial(K)$.

COROLlaRY 3.4. Let $K$ be a surface isotropic convex body in $\mathbf{R}^{n}$. Then

$$
\left(\frac{\partial(K)}{n}\right)^{n} \leqslant|\Pi K| \leqslant \omega_{n}\left(\frac{\omega_{n-1}}{n \omega_{n}}\right)^{n} \partial(K)^{n}
$$

Note that all the estimates in Proposition 3.3 and Corollary 3.4 are sharp: it is enough to consider the ball and the cube.

Proposition 3.3 has some immediate consequences. Assume that $|K|=1$ and $\partial(K)=\partial_{K}$. We have

$$
\int_{S^{n-1}} \frac{1}{\left|P_{\theta}(K)\right|^{n}} \sigma(d \theta)=\frac{\left|\Pi^{*} K\right|}{\omega_{n}} \leqslant\left(\frac{c \sqrt{n}}{\partial_{K}}\right)^{n}
$$

hence Markov's inequality gives.

THEOREM 3.5. There exists an absolute constant $c>0$, such that for every surface isotropic body $K$ of volume 1 ,

$$
c \frac{\partial_{K}}{\sqrt{n}} \leqslant\left|P_{\theta}(K)\right| \leqslant \frac{\partial_{K}}{2 \sqrt{n}}
$$

holds true for all $\theta$ in a subset of $S^{n-1}$ with $\sigma$-measure exceeding $1-2^{-n}$. 
It is an observation of C. M. Petty [6] that, if $T$ is a linear operator on $\mathbf{R}^{n}$ of determinant 1 , then, for every $K$,

$$
\Pi^{*}(T K)=T\left(\Pi^{*} K\right) \text {. }
$$

In particular, the volume of the polar projection body is invariant under volume preserving affine transformations. Then, Proposition 3.3 and Corollary 3.4 show that $\left|\Pi^{*} K\right|^{1 / n}$ and $|\Pi K|^{1 / n}$ are determined up to absolute constants by the minimal surface area $\partial_{K}$.

THEOREM 3.6. There exist absolute constants $c_{1}, c_{2}, c_{3}, c_{4}>0$ such that, if $|K|=1$, then

$$
\frac{c_{1}}{\partial_{K}} \leqslant\left|\Pi^{*} K\right|^{1 / n} \leqslant \frac{c_{2}}{\partial_{K}} . \quad \text { and } \quad c_{3} \frac{\partial_{K}}{n} \leqslant|\Pi K|^{1 / n} \leqslant c_{4} \frac{\partial_{K}}{n} .
$$

G. Zhang [11] has proved that, if $|K|=1$, then

$$
\left|\Pi^{*} K\right| \geqslant\left(\begin{array}{c}
2 n \\
n
\end{array}\right) n^{-n}
$$

with equality if, and only if, $K$ is a simplex. This can be combined with Proposition 3.3 to give an upper estimate for $\partial_{K}$.

THeOREm 3.7. For every convex body $K$ we have

$$
\partial_{K} \leqslant 4 n^{2} /\left[n !\left(\begin{array}{c}
2 n \\
n
\end{array}\right)\right]^{1 / n} \leqslant c n,
$$

where $c>0$ is an absolute constant.

The constant $c$ may be (asymptotically) chosen to be equal to $e$. K. Ball [2] has proved an exact reverse isoperimetric inequality of this type in the symmetric case. Let $Q$ be the cube of volume 1 . Every symmetric convex body $K$ has an affine image $\tilde{K}$ satisfying $|\tilde{K}|=1$ and $\partial(\tilde{K}) \leqslant \partial(Q)$. In our notation, this means that

$$
\partial_{K} \leqslant 2 n
$$

for every symmetric convex body. Observe that this would follow from (3.6) if the cube was minimizing $\left|\Pi^{*} K\right|$ over all symmetric bodies of volume 1 . Combining (3.11) with Proposition 3.1 and dropping the assumption that $|K|=$ 1 we get.

Proposition 3.8. Let $K$ be a symmetric convex body in $\mathbf{R}^{n}$ whose area measure $\sigma_{K}$ is isotropic. Then

$$
\left|P_{\theta}(K)\right| \leqslant \sqrt{n}|K|^{(n-1) / n}
$$

for every $\theta \in S^{n-1}$.

The estimate given by Proposition 3.8 was conjectured by $\mathrm{K}$. Ball, and it is sharp for the cube. One can use Ball's non-symmetric reverse isoperimetric 
inequality (or Theorem 3.7) to state an analogous result for an arbitrary surface isotropic body $K$.

We now turn to lower bounds. We shall make use of an extension of the Loomis-Whitney inequality that $\mathrm{K}$. Ball [1] proved and used for the proof of (1.5):

LemMa 3.9. Let $K$ be a convex body in $\mathbf{R}^{n}$, let $\left\{u_{j}\right\}_{j \leqslant n}$ be a sequence of unit vectors, and let $\left\{c_{j}\right\}_{j \leqslant m}$ be a sequence of positive numbers. Suppose that

$$
\mathrm{Id}=\sum_{j=1}^{m} c_{j} u_{j} \otimes u_{j}
$$

Then

$$
|K|^{n-1} \leqslant \sum_{j=1}^{m}\left|P_{u j}(K)\right|^{c_{j}}
$$

Assume that $K$ is a polytope with facets $F_{j}$ and normals $u_{j}$, for $j=1, \ldots, m$, which has isotropic area measure. Because of (3.4) we can apply Lemma 3.9 to obtain

$$
(n-1) \log |K| \leqslant \frac{n}{\partial(K)} \sum_{j=1}^{m} \log \left|P_{u_{j}}(K)\right|\left|F_{j}\right|,
$$

which can be written in the form

$$
|K|^{(n-1) / n} \leqslant \exp \left(\frac{1}{\partial(K)} \sum_{j=1}^{m} \log \left|P_{u_{j}}(K)\right|\left|F_{j}\right|\right) .
$$

Using this fact and an approximation argument, we can prove the following.

Proposition 3.10. Let $K$ be a convex body in $\mathbf{R}^{n}$ whose area measure $\sigma_{K}$ is isotropic. Then

$$
|K|^{(n-1) / n} \leqslant \exp \left(\frac{1}{\partial(K)} \int_{S^{n-1}} \log \left|P_{\theta}(K)\right| \sigma_{K}(d \theta)\right) .
$$

Proof. Assume that $\left\{K_{i}\right\}$ is a sequence of polytopes, with $\sigma_{K_{i}}$ isotropic and $K_{i} \rightarrow K$. By (3.12) we have

$$
\left|K_{i}\right|^{(n-1) / n} \leqslant \exp \left(\frac{1}{\partial\left(K_{i}\right)} \int_{S^{n-1}} \log \left|P_{\theta}\left(K_{i}\right)\right| \sigma_{K_{i}}(d \theta)\right)
$$

for every $i$, and taking limit as $i \rightarrow \infty$ we conclude the proof.

Combining Cauchy's formula with the isoperimetric inequality and Ball's reverse isoperimetric inequality, we have 
Proposition 3.11. Let $K$ be a surface isotropic symmetric convex body in $\mathbf{R}^{n}$. Then

$$
\max _{\theta}\left|P_{\theta}(K)\right| \geqslant\left[\omega_{n-1} / \omega_{n}^{(n-1) / n}\right]|K|^{(n-1) / n} \geqslant c|K|^{(n-1) / n}
$$

and

$$
\min _{\theta}\left|P_{\theta}(K)\right| \leqslant\left[2 \omega_{n-1} / \omega_{n}\right]|K|^{(n-1) / n} \leqslant c \sqrt{n}|K|^{(n-1) / n}
$$

The first inequality is sharp for a ball: the ball of volume 1 has all its projections of volume of the order of 1 . We now show that the second inequality is also exact up to an absolute constant.

Proposition 3.12. There exists a surface isotropic symmetric convex body. such that

$$
c \sqrt{n}|K|^{(n-1) / n} \leqslant\left|P_{\theta}(K)\right| \leqslant \sqrt{n}|K|^{(n-1) / n}
$$

for every $\theta \in S^{n-1}$.

Proof. We shall follow Ball's counter-example to Shephard's problem [1]. What we want to make sure is that the example will be surface isotropic.

Let $\left\{e_{j}: j \leqslant n\right\}$ be an orthonormal basis in $\mathbb{R}^{n}$. It is a known fact going back to the work of Kashin (see [7, Chapter 6]) that there exists an orthogonal transformation $T \in O(n)$ such that

$$
c_{1} \sqrt{n}|x| \leqslant\|x\|_{1}+\|\dot{T} x\|_{1} \leqslant c_{2} \sqrt{n}|x|
$$

for every $x \in \mathbf{R}^{n}$, where $\|x\|_{1}=\sum_{j=1}^{n}\left|\left\langle x, e_{j}\right\rangle\right|$. It is clear that the sum of two isotropic measures on $S^{n-1}$ is isotropic; therefore the measure

$$
\sigma=\sigma_{Q}+\sigma_{T^{-1} Q}
$$

is isotropic. From Minkowski's existence theorem, there exists a symmetric polytope $K$ with $\sigma_{K}=\sigma$. Then $K$ has normals $\pm e_{j}, \pm T^{*} e_{j}$, and the area of each facet of $K$ is equal to 1. Using (3.13) we get

$$
\left|P_{\theta}(K)\right|=\sum_{j=1}^{n}\left(\left|\left\langle\theta, e_{j}\right\rangle\right|+\left|\left\langle\theta, T^{*} e_{j}\right\rangle\right|\right)=\|\theta\|_{1}+\|T \theta\|_{1} \geqslant c_{1} \sqrt{n}
$$

for every $\theta \in S^{n-1}$. As in [1], consider the body

$$
L=\left\{x \in \mathbf{R}^{n}:\left|\left\langle x, e_{j}\right\rangle\right| \leqslant 1,\left|\left\langle x, T^{*} e_{j}\right\rangle\right| \leqslant 1, j=1, \ldots, n\right\} .
$$

By a result of Vaaler $[\mathbf{1 0}],|L|^{1 / n} \geqslant \sqrt{2}$, and by Minkowski's inequality,

$$
|K|^{(n-1) / n}|L|^{1 / n} \leqslant \frac{1}{n} \int_{S^{n-1}} h_{L}(u) \sigma_{K}(d u)=\frac{2}{n} \sum_{j=1}^{n}\left(h_{L}\left(e_{j}\right)+h_{L}\left(T^{*} e_{j}\right)\right) \leqslant 4 .
$$


It follows that

$$
\left|P_{\theta}(K)\right| \geqslant \frac{c_{1}}{4} \sqrt{n}|K|^{(n-1) / n}|L|^{1 / n} \geqslant c \sqrt{n}|K|^{(n-1) / n},
$$

which completes the proof.

COROLlary 3.13. There exist two surface isotropic symmetric convex bodies $K_{1}$ and $K_{2}$ in $\mathbf{R}^{n}$ such that $\left|P_{\theta}\left(K_{1}\right)\right| \leqslant\left|P_{\theta}\left(K_{2}\right)\right|$ for every $\theta \in S^{n-1}$, but $\left|K_{1}\right| \geqslant c \sqrt{n}\left|K_{2}\right|$, where $c>0$ is an absolute constant.

\$4. Stability of the surface isotropic position. Let $K$ be a convex body in $\mathbf{R}^{n}$ with volume $|K|=1$. Assume that it is close to the surface isotropic position, in the sense that all the integrals

$$
\int_{S^{n-1}}\langle u, \theta\rangle^{2} \sigma_{K}(d u), \quad \theta \in S^{n-1},
$$

are equivalent up to some constant $\beta>1$. We shall show that the surface area $\partial(K)$ of $K$ is close to the minimal surface area $\partial_{K}$. More precisely, we have the following stability result.

TheOREM 4.1. Let $K$ be a convex body in $\mathbf{R}^{n}$ with $|K|=1$. Assume that there exist $A>0$ and $\beta>1$ such that

$$
A \leqslant \int_{S^{n-1}}\langle u, \theta\rangle^{2} \sigma_{K}(d u) \leqslant \beta A
$$

for every $\theta \in S^{n-1}$. Then,

$$
\partial_{K} \leqslant \partial(K) \leqslant c \beta \partial_{K}
$$

where $c>0$ is an absolute constant.

Proof. Choosing $\theta=e_{i}$ for $i=1, \ldots, n$ in (4.1) and adding all inequalities we get

$$
\frac{1}{\beta} \frac{\partial(K)}{n} \leqslant \int_{S^{n+1}}\langle u, \theta\rangle^{2} \sigma_{K}(d u) \leqslant \beta \frac{\partial(K)}{n}, \quad \theta \in S^{n-1}
$$

We may assume that $K$ is a polytope with facets $F_{j}$ and normals $u_{j}$ for $j=$ $1, \ldots, m$. Consider the ellipsoid $E$ defined by

$$
\|x\|_{E}^{2}=\sum_{j=1}^{m}\left|F_{j}\right|\left\langle x, u_{j}\right\rangle^{2}=\int_{S^{n-1}}\langle u, x\rangle^{2} \sigma_{K}(d u) .
$$


Then $E=T D_{n}$ for some linear transformation $T$, and (4.3) shows that

$$
\frac{1}{\beta} \frac{\partial(K)}{n}|T x|^{2} \leqslant|x|^{2}=\|T x\|_{E}^{2}=\sum_{j=1}^{m}\left|F_{j}\right|\left\langle x, T^{*}\left(u_{j}\right)\right\rangle^{2} \leqslant \beta \frac{\partial(K)}{n}|T x|^{2} .
$$

In particular,

$$
|\operatorname{det} T|^{1 / n} \leqslant\left\|T: l_{2}^{n} \rightarrow l_{2}^{n}\right\| \leqslant \sqrt{\beta n / \partial(K)} .
$$

Consider the body $K_{1}=T^{-1} K$. It has normals $u_{j}^{\prime}=T^{*}\left(u_{j}\right) /\left|T^{*}\left(u_{j}\right)\right|$ and facet areas $\left|F_{j}^{\prime}\right|=\left|T^{*}\left(u_{j}\right)\right|\left|F_{j}\right| / \mid$ det $T \mid$. From (4.5), it is clear that

$$
|x|^{2}=\sum_{j=1}^{m}\left|F_{j}\right|\left|T^{*}\left(u_{j}\right)\right|^{2}\left\langle x, u_{j}^{\prime}\right\rangle^{2},
$$

which implies

$$
\mathrm{Id}=\sum_{j=1}^{m}\left|F_{j}\right|\left|T^{*}\left(u_{j}\right)\right|^{2} u_{j}^{\prime} \otimes u_{j}^{\prime} .
$$

Consider the polar projection body of $K_{1}$. We have

$$
\|\theta\|_{\Pi^{*} K_{1}}=\left|P_{\theta}\left(K_{1}\right)\right|=\sum_{j=1}^{m} \frac{\left|F_{j}\right|\left|T^{*}\left(u_{j}\right)\right|}{2|\operatorname{det} T|}\left|\left\langle\theta, u_{j}^{\prime}\right\rangle\right|
$$

and Lemma 3.2 shows that

$$
\begin{aligned}
\left|\Pi^{*} K_{1}\right| & \leqslant \frac{2^{n}}{n !} \prod_{j=1}^{m}\left(2\left|T^{*}\left(u_{j}\right)\right||\operatorname{det} T|\right)^{\left|F_{j}\right|\left|T^{*}\left(u_{j}\right)\right|^{2}} \\
& \leqslant \frac{2^{n}}{n !}(2\|T\||\operatorname{det} T|)^{\Sigma_{j}\left|F_{j}\right|\left|T^{*}\left(u_{j}\right)\right|^{2}} \\
& =\frac{4^{n}}{n !}\|T\|^{n}|\operatorname{det} T|^{n} .
\end{aligned}
$$

Using Theorem 3.6 and (4.6), we see that

$$
\frac{c}{\partial_{K}} \leqslant\left|K_{1}\right|^{(n-1) / n}\left|\Pi^{*} K_{1}\right|^{1 / n} \leqslant \frac{4}{(n !)^{1 / n}}\|T\||\operatorname{det} T|^{1 / n} \leqslant \frac{4 e \beta}{\partial(K)},
$$

which shows that

$$
\partial_{K} \leqslant \partial(K) \leqslant \frac{4 e}{c} \beta \partial_{K}
$$

\section{References}

1. K. M. Ball. Shadows of convex bodies. Trans. Amer. Math. Soc., 327 (1991), $891-901$.

2. K. M. Ball. Volume ratios and a reverse isoperimetric inequality. J. London Math. Soc. (2), 44 (1991), 351-359.

3. Y. Gordon, M. Meyer and S. Reisner. Zonoids with minimal volume product: A new proof. Proc. Amer. Math. Soc., 104 (1988), 273-276.

4. V. D. Milman and A. Pajor. Isotropic position and inertia ellipsoids and zonoids of the unit ball of a normed $n$-dimensional space. Lecture Notes in Mathematics 1376 (Springer, 1989), 64-104. 
5. C. M. Petty. Surface area of a convex body under affine transformations. Proc. Amer. Math. Soc., 12 (1961), 824-828.

6. C. M. Petty. Projection bodies. Proc. Colloq. on Convexity, Copenhagen, 1967, 234-241.

7. G. Pisier. The Volume of Convex Bodies and Banach Space Geometry (Cambridge University Press, 1989).

8. S. Reisner. Zonoids with minimal volume product. Math. Z., 192 (1986), 339-346.

9. R. Schneider. Convex Bodies: The Brunn-Minkowski Theory (Cambridge University Press, 1993).

10. J. D. Vaaler. A geometric inequality with applications to linear forms. Pacific J. Math., 83 (1979), 543-553.

11. G. Zhang. Restricted chord projection and affine inequalities. Geom. Dedicata, 39 (1991), 213 222.

Professor A. Giannopoulos,

Department of Mathematics,

University of Crete,

Iraklion,

Crete,

Greece.

e-mail : deligia $(a$ talos.cc.uch.gr

Professor M. Papadimitrakis,

Department of Mathematics,

University of Crete,

Iraklion,

Crete,

Greece.

e-mail : papadim@talos.cc.uch.gr
52A40: CONVEX AND DISCRETE GEOMETRY; General convexity; Inequalities and extreme problems.

Received on the 15th of December, 1997. 